\title{
Influência da cor verde do matapi PET na captura de camarões do gênero Macrobrachium Bate, 1888 em viveiros de piscicultura no município do Cantá, Roraima, Brasil.
}

\author{
Ronilson Moura Cavalcante ${ }^{1 *}$, Sandro Loris Aquino-Pereira ${ }^{2} \&$ Muara Santana do \\ Nascimento $^{1}$
}

\begin{abstract}
${ }^{1}$ Universidade Estadual de Roraima (UERR). Rua Sete de Setembro 231, Canarinho CEP 69306-530C, Boa Vista, RR, Brasil. Programa de Pós-graduação em Agroecologia (PPGA)/Universidade Estadual de Roraima (UERR)/ Embrapa Roraima/IFRR.

${ }^{2}$ Empresa Brasileira de Pesquisa Agropecuária - Embrapa Roraima. Rodovia BR-174, Km 8, Distrito Industrial, Boa Vista-RR. E-mail: sandro.loris@embrapa.br.
\end{abstract}

*E-mail para correspondência: ronilsonmouracavalcante@gmail.com

Recebido em:21 de dezembro de 2016. Aceito em:07 de fevereiro de 2017. Publicado PDF em:10 de março de 2017

\section{RESUMO}

Influência da cor verde do matapi PET na captura de camarões do gênero Macrobrachium Bate, 1888 em viveiros de piscicultura no município do Cantá, Roraima, Brasil. Armadilhas tipo matapis confeccionadas a partir de garrafas PET foram utilizadas na captura de camarões do gênero Macrobrachium em viveiros de piscicultura para avaliar a influência e eficiência da cor verde e transparente dos matapis na captura dos camarões. Foram capturados 1.308 camarões, das espécies Macrobrachium amazonicum e $M$. jelski, com comprimento médio de $35 \pm 4 \mathrm{~mm}$. As armadilhas matapi PET de cor verde foram mais eficientes na captura de camarões, do que as armadilhas matapi PET transparentes com a mesma Captura por Unidade de Esforço (CPUE).

PALAVRAS CHAVE: Crustacea, amostragem, camarão regional, piscicultura, Roraima.

\section{ABSTRACT}

Influence of green color in trap PET (matapi) in cattle catch of shrimps Macrobrachium Bate, 1888 in fishponds in the fish farm in Cantá county, Roraima, Brazil. Matapis type traps made from PET bottles were used to catch Macrobrachium shrimps in fish farms to evaluate the influence and efficiency of green and transparent matapis on shrimp trawling. A total of 1.308 prawns of the species Macrobrachium amazonicum and M. jelski were caught, with an average length of $35 \pm 4 \mathrm{~mm}$. Green matapi PET traps were more efficient in shrimp capture than were transparent PET matapi traps with the same catch per unit effort (CPUE).

KEY WORDS: Crustacea, sampling, regional shrimp, fish farming, Roraima.

\section{INTRODUÇÃO}

Em Roraima é registrada a ocorrência de quatro gêneros da Família Palaemonidae Rafinesque, 1815, sendo eles: Euryrhynchus; Macrobrachium Bate, 1888; Palaemonetes e Pseudopalaemon. Dentre as espécies de Macrobrachium são registradas no Estado: $M$. amazonicum (Heller 1862); M. brasiliense (Heller 1862); M. heterochirus (Wiegmann 1836); M. holthuisi Genofre \& Lobão 1978; M. inpa Kensley \& Walker 1982; M. jelskii (Miers
1877); M. nattereri (Heller 1862); e três morfotipos de Macrobrachium (Guterres et al. 2010; Castro 2012; Lima et al. 2012; Castro \& Silva 2013; Aquino-Pereira et al. 2013).

A carcinicultura de água doce, realizada com o gênero Macrobrachium tem sido reconhecida como uma forma de produzir crustáceos com baixo impacto ambiental (New et al. 2000). Adapta-se muito bem aos sistemas familiares de produção e atende aos preceitos da aqüicultura sustentável. Os índices econômicos obtidos até o presente atestam de forma inequívoca a 
viabilidade econômica da atividade (Valenti 2002).

Em Roraima, alguns estudos foram realizados para a melhor compreensão sobre a biologia reprodutiva e cultivo de espécies de Macrobrachium. Lima \& Castro (2012) ao estudar a reprodução de $M$. amazonicum em viveiros de piscicultura na região do Apiaú (município de Mucajaí, Roraima) verificaram a presença de todas as fases de vida da espécie e que esta se reproduz $o$ ano inteiro. Isso contribuiu para Cavalcante \& Castro (2014) que observaram que a espécie de camarão de água doce, $M$. jelskii, está altamente adaptadas aos viveiros de engorda de tambaqui, em Roraima e conseguem fechar seu ciclo de reprodução, uma vez que foram constatados camarões de todas as classes de tamanho desde a pós-larva, adultos e fêmeas ovígeras nas coletas realizadas nos viveiros.

A captura de camarões é feita utilizando-se redes de arrasto e armadilhas fixas, conhecidas como "matapis". O matapi tem se mostrado mais econômico e ambientalmente favorável para este fim, pois além de minimizar a captura de fauna acompanhante, promove menor gasto de tempo e energia nas capturas (Vieira 2003). Os matapis utilizados pela maioria dos ribeirinhos da Amazônia não são seletivos e promovem a captura de camarões imaturos ou em fase inicial de maturação, fato este que pode comprometer, juntamente com a sobreexploração, a renovação dos estoques naturais na região (Lima 2014). Além disso, o matapi é construído a partir da palmeira jupati (Raphia vinifer) e tem vida útil média de apenas três meses (Camargo et al. 2009).

Fouilland \& Fossati (1996) testaram a eficiência de captura de garrafas de plástico (garrafas PET) como armadilhas para avaliação da população de Macrobrachium nos rios Nuku -Hiva (Ilhas Marquesas, Polinésia Francesa) e mostraram que estas têm uma eficiência de amostragem entre 40 e $47 \%$ para uma densidade de 8,3 armadilhas por $\mathrm{m}^{2}$. Posteriormente, testaram também os efeitos de alguns fatores operacionais (direção, cor, isca e lastro) na amostragem de Macrobrachium utilizando estas pequenas armadilhas feitas com garrafas plásticas de água mineral (garrafas PET) obtendo ótimos resultados para o fator operacional de direção (Fouilland \& Fossati 1998). Camargo et al. (2009) também testaram o uso da armadilha matapi, usando garrafas PET (politereftalato de etileno) recicladas para captura de camarões e mostraram que são mais seletivos e que capturam exemplares com comprimento médio e o mínimo maiores que os capturados pelo matapi de jupati. Ainda segundo Fouilland \& Fossati (1996), as armadilhas de garrafas PET são pequenos dispositivos de amostragem, mas é possível usar um grande número deles. Estes materiais são baratos, fáceis de usar por um único pesquisador, pescador ou produtor em locais distantes com acesso difícil (pois apesar de volumosas, são leves) e têm uma seletividade constante. Além disso, seu uso não prejudica os crustáceos e respeita o meio ambiente.

Segundo Volpato et al. (2004), a discriminação ambiental torna-se útil para indicar a percepção do animal e o estímulo específico que torna um ambiente mais atraente do que outros quando oferecidas opções para a escolha, pode ser um indicativo de condições de bem-estar para o animal, conferindo possíveis vantagens na alimentação, migração ou reprodução. Somado a isso, Luchiari \& Pirhonen (2008) afirmam que o conhecimento no tocante aos efeitos de cores sobre animais aquáticos cultivados ainda é muito restrito; e estudos dos efeitos de coloração ambiental podem contribuir para melhorar o ambiente de cultivo e assim aumentar a produtividade.

Apesar disso, os efeitos relacionados não são semelhantes para todas as espécies, e até mesmo o inverso pode ser demonstrado, indicando um efeito espécie-específico. Assim, isso ilustra a necessidade de determinar as preferências de cor para cada espécie. Neste contexto, o presente estudo teve como objetivo avaliar a influência da cor verde e transparente de armadilhas tipo matapi confeccionadas de garrafas PET na captura de camarões do gênero Macrobrachium.

\section{MATERIAL E MÉTODOS}

\section{Área de estudo}

A pesquisa foi realizada na Agropecuária Carvalho, fazenda "2 irmãos", localizada na vicinal 02 do município do Cantá (Roraima, Brasil, $\left.\quad 2^{\circ} 28^{\prime} 20,24^{\prime \prime} \mathrm{N} \quad 60^{\circ} 35^{\prime} 02,39^{\prime} \mathrm{O}\right)$, compreendendo a infraestrutura da piscicultura na propriedade (Figura 1). A propriedade é constituída por setenta e três viveiros escavados de fundo natural com disposição sequencial onde se pratica a criação semi-intensiva de tambaqui (Colossoma macropomum Cuvier 1818) para fins comerciais, onde são 


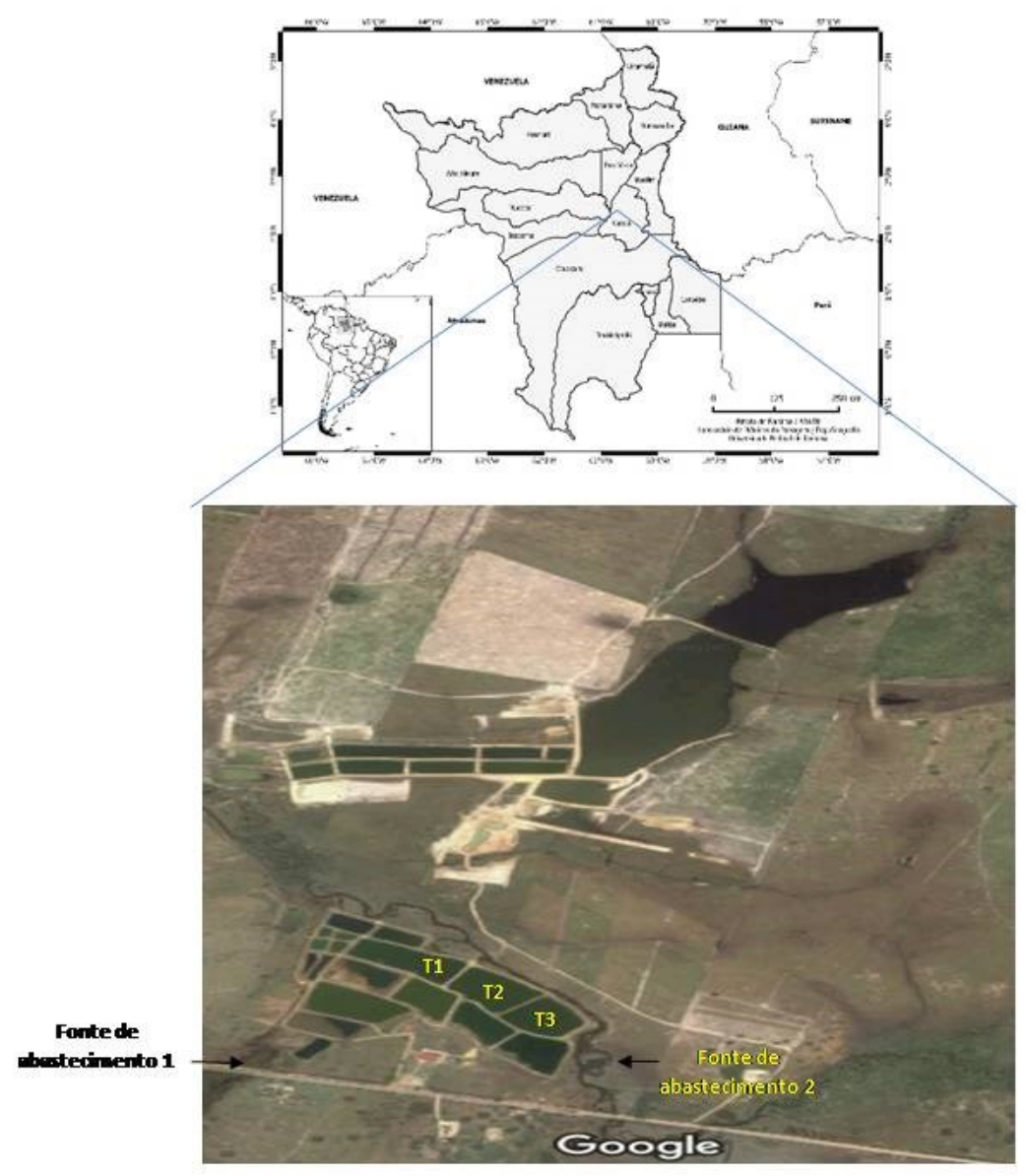

Figura 1. Viveiros amostrados, fontes de abastecimento de água e localização da piscicultura Agropecuária Carvalho, no município do Cantá-RR. Fonte: GOOGLE EARTH (2016).

encontrados camarões invasores nos viveiros de tambaqui. Foram utilizados três viveiros de engorda, com área média de espelho d'água de 0,97ha, profundidade média de $1,70 \mathrm{~m}$ e área total de $29.237 \mathrm{~m}^{2}$. Estes são abastecidos por dois corpos d'água (fontes de abastecimento) naturais que passam pela propriedade e são as fontes das invasões dos camarões nos viveiros.

\section{Confecção da armadilha matapi de garrafa PET}

Para capturar os camarões foram confeccionados armadilhas matapi de garrafa PET, da seguinte forma: duas garrafas PET padronizada de dois litros e lisas, devidamente limpas e das mesmas cores transparentes e verdes. Em seguida, as garrafas foram cortadas para fazer o corpo do matapi PET que possui cerca de $32 \mathrm{~cm}$ de comprimento por $10 \mathrm{~cm}$ de diâmetro, sua abertura nas entradas laterais são de $2 \mathrm{~cm}$. As laterais da gaiola são formadas por uma espécie de funil (boca das garrafas invertidas), que facilita a entrada dos camarões, mas impede a saída dos animais maiores. Após isso, foram perfuradas com furador $\mathrm{e}$ posteriormente encaixadas com o corpo e amarradas com fio de náilon; e ainda, foram presos dois pesos de chumbo para o lastro, na parte externa da armadilha (Figura 2).

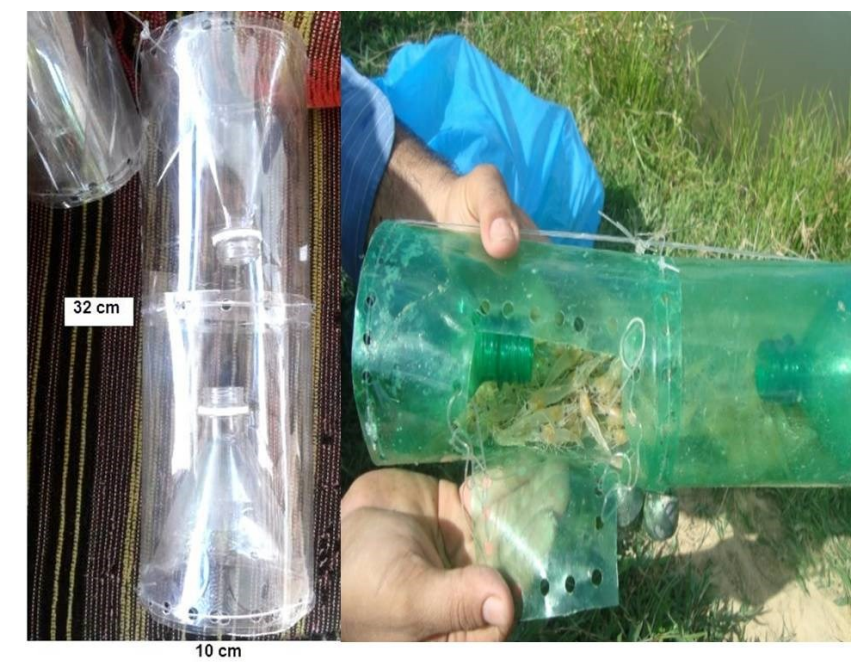

Figura 2. Exemplares das armadilhas matapi PET na cor verde e transparente. 


\section{Procedimento de amostragem}

Foram realizadas duas amostragens, em seis (06) viveiros de criação de tambaqui, entre os meses de junho e julho de 2015 para a amostragem dos camarões. Disto resultaram 18 repetições, pois as duas coletas foram somadas para medir a eficiência da cor do matapi PET na captura dos camarões, e não o efeito temporal. Como iscas foram utilizadas peletes de ração comercial para peixe e colocadas nas armadilhas matapis. Em cada viveiro dispôs-se de seis (06) matapis PET intercalados em cada margem dos viveiros, sendo três (03) verdes e três (03) transparentes (Figura 2). Todas as amostragens ocorreram no período vespertino (13h) e tiveram um período de espera de uma hora para retirada dos matapis PET da água. Os matapis PET foram dispostos nas duas margens dos viveiros da criação do tambaqui, sendo o primeiro e terceiro matapis distantes cinco (05) metros da margem e a distância aproximada de sessenta (60) metros do terceiro matapi, ao meio. Em uma das margens foram disposto dois (02) matapis verdes e um (01) transparente; e na outra margem forma dispostos dois (02) matapis transparentes e um (01) verde; variando a profundidade $(1,5$ a $1,7 \mathrm{~m})$ de acordo com o declive do viveiro (Figura 3).

Posteriormente, os camarões foram contabilizados e acondicionados em fracos de vidro com álcool $70 \%$, individualizados por matapi e etiquetados; sendo que a informação de cada matapi foi registrada em caderno de campo. Todo o material foi transportado para o Laboratório de Organismos Aquáticos da Amazônia (LOAM) da Embrapa Roraima para posteriores análises e identificação. Desta forma, no LOAM, procedeu-se a medição do comprimento do cefalotórax de cada um dos indivíduos capturados. Todos os dados foram digitados em uma tabela de dados para o cálculo da Captura por Unidade de Esforço (CPUE). Assim, utilizou-se a razão entre o número de indivíduos capturados $(\mathrm{N})$ pelo tempo de submersão em horas $(\mathrm{t})$, segundo a fórmula:

$$
\mathrm{CPUE}=(\mathrm{N} / \mathrm{t})
$$

Os dados da CPUE de ambos matapi PET foram avaliados utilizando-se o programa Bioestat 5.0 (Ayres et al. 2007). E, para testar as médias da CPUE dos matapis, foi aplicado um teste $t$ com um nível de significância de 5\% (Zar 2010). Todo o material testemunho esta depositado na Coleção científica do Laboratório de Organismos Aquáticos da Amazônia (LOAM) e parte deste material também será depositado na Coleção de Invertebrados do Museu Integrado de Roraima (MIRR).

\section{RESULTADOS E DISCUSSÃO}

Um total de 1.308 camarões foi capturado, com comprimento médio de $35 \pm 4 \mathrm{~mm}$, sendo que 494 foram capturados nos matapis PET transparentes e 814 nos matapis PET verdes.
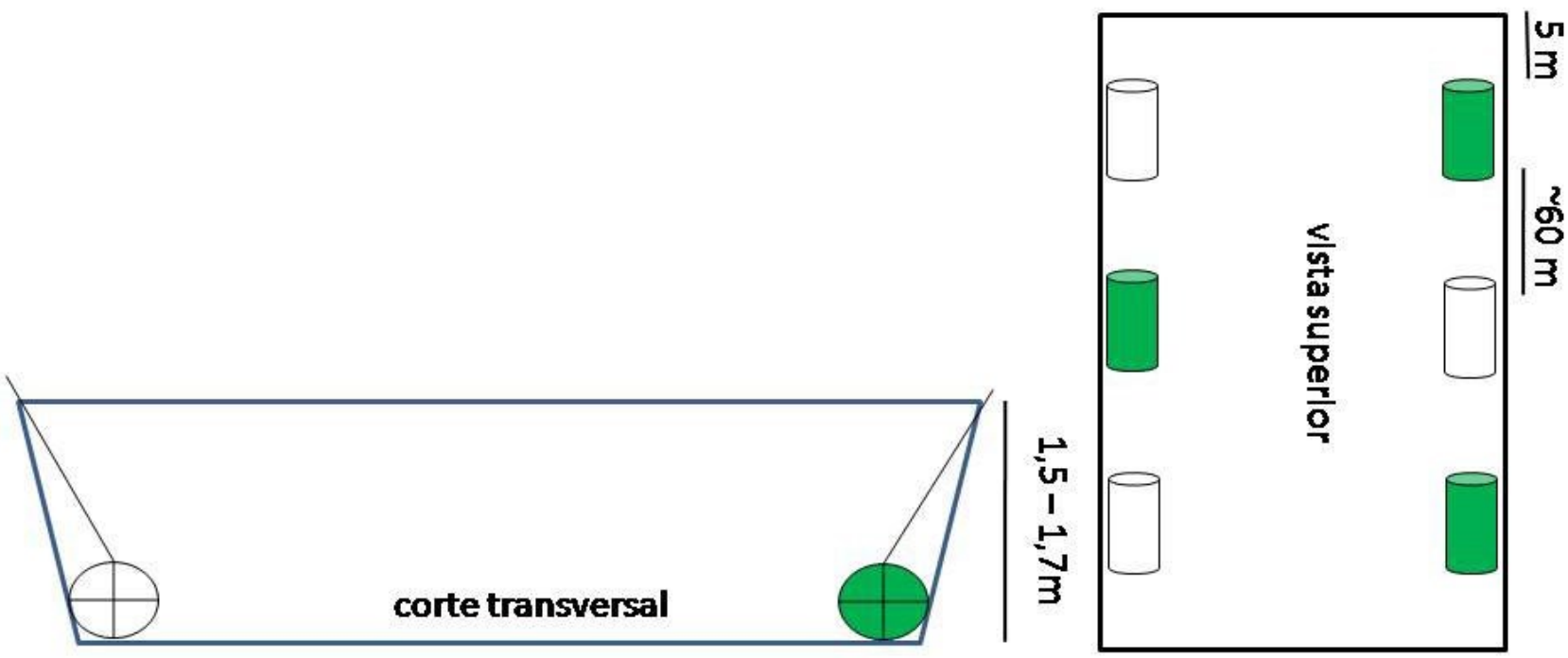

Figura 3. Esquema da disposição dos matapis pet ao longo das margens dos viveiros. 
Destes foram identificadas duas espécies de camarões ocorrendo nos viveiros desta piscicultura, o Macrobrachium amazonicum e M. jelski. A Tabela 1 apresenta as quantidades de camarões capturadas pelas armadilhas de matapi PET (transparentes e verdes). O teste $t$ de comparação de médias de CPUE dos matapis PET detectou diferenças significativas $(t=2,65 ; p=0,02)$ entre os matapis de diferentes cores. E os resultados mostraram que o matapi PET verde capturou mais indivíduos que o matapi PET transparente (Figura 4).

Como descrito por Marshall et al. (1996) muitas espécies de crustáceos têm a capacidade de visão em cores, fornecendo uma vantagem evolutiva em habitats onde a luz é composta de muitas cores. Desta forma, a percepção de cores é um recurso útil para os animais reconhecerem os detalhes do seu ambiente, como: abrigo, alimento, fêmeas e etc. (Luchiari et al. 2012). Alguns estudos têm demonstrado a influência da cor em processos biológicos de crustáceos e outros animais aquáticos (Mariappan \& Balasundaram 1999; Volpato et al. 2004; Luchiari et al. 2008; Luchiari et al. 2012).

Mariappan \& Balasundaram (1999) observaram que a cor e a textura do abrigo influenciaram no comportamento de entocamento de $M$. nobilli (Henderson \& Matthai 1910) (juvenis, machos e fêmeas) e verificaram que está espécie evita abrigos transparentes e que jovens e adultos apresentam uma preferência por abrigos de cor escura (preto). Santos (2013) analisou o efeito da coloração do abrigo sobre a frequência de ocupação e das atividades comportamentais de camarões $M$. rosenbergii (De Man 1879) em cultivos misto, monosexo macho e monosexo fêmea; e concluíram que está espécie quando submetidos aos cultivos mistos e monosexo macho apresentam uma tendência a preferência por abrigo de cor preta e em cultivo monosexo fêmea uma tendência pela utilização de abrigos de cor vermelha e laranja. Estes trabalhos corroboram o resultado encontrado neste trabalho, pois as armadilhas matapis PET podem ser considerados abrigos temporários apesar de possuírem iscas para atração dos camarões.

Opostamente, Fouilland \& Fossati (1998) testaram os efeitos de alguns fatores operacionais na amostragem de Macrobrachium utilizando pequenas armadilhas feitas com garrafas plásticas de água mineral, dentre estes a cor; e o número de camarões capturados em armadilhas pintadas não diferiu daqueles capturados nas amostras transparentes. Assim, vários trabalhos têm relatado a influência de cores diferentes em diferentes fases de vida dos camarões e em diferentes parâmetros (coloração do tanque, dos abrigos, do substrato, de armadilhas ou da iluminação) utilizadas por estes para o seu bem -estar, tanto na natureza quanto em cativeiro (Lin \& Omori 1993; Fouilland \& Fossati 1998; Mariappan \& Balasundaram 1999; Meager et al. 2005; Yasharian et al. 2005; Luchiari \& Pirhonen 2008; Luchiari et al. 2009; Luchiari et al. 2012).

Tabela 1. Total de camarões amostrados nos três viveiros escavados utilizando matapis PET verde e transparente (Coleta 1: 24/06/2015; Coleta 2: 08/07/2015).

Matapis PET

\begin{tabular}{c|c|c|c|c|c|c} 
Coleta 1 & \multicolumn{3}{c}{ Verde } & \multicolumn{3}{c}{ Transparente } \\
\hline Viveiros & $\mathbf{1}$ & $\mathbf{2}$ & $\mathbf{3}$ & $\mathbf{1}$ & $\mathbf{2}$ & $\mathbf{3}$ \\
\hline $\mathbf{1}$ & 116 & 36 & 16 & 59 & 17 & 28 \\
\hline $\mathbf{2}$ & 37 & 11 & 19 & 22 & 16 & 20 \\
\hline $\mathbf{3}$ & 96 & 39 & 28 & 35 & 22 & 27 \\
\hline
\end{tabular}

\begin{tabular}{c|c|c|c|c|c|c} 
Coleta 2 & \multicolumn{3}{c}{ Verde } & \multicolumn{4}{c}{ Transparente } \\
\hline Viveiros & $\mathbf{1}$ & $\mathbf{2}$ & $\mathbf{3}$ & $\mathbf{1}$ & $\mathbf{2}$ & $\mathbf{3}$ \\
\hline $\mathbf{1}$ & 48 & 120 & 34 & 40 & 25 & 33 \\
\hline $\mathbf{2}$ & 41 & 21 & 32 & 9 & 24 & 16 \\
\hline $\mathbf{3}$ & 70 & 28 & 22 & 39 & 28 & 34 \\
\hline
\end{tabular}




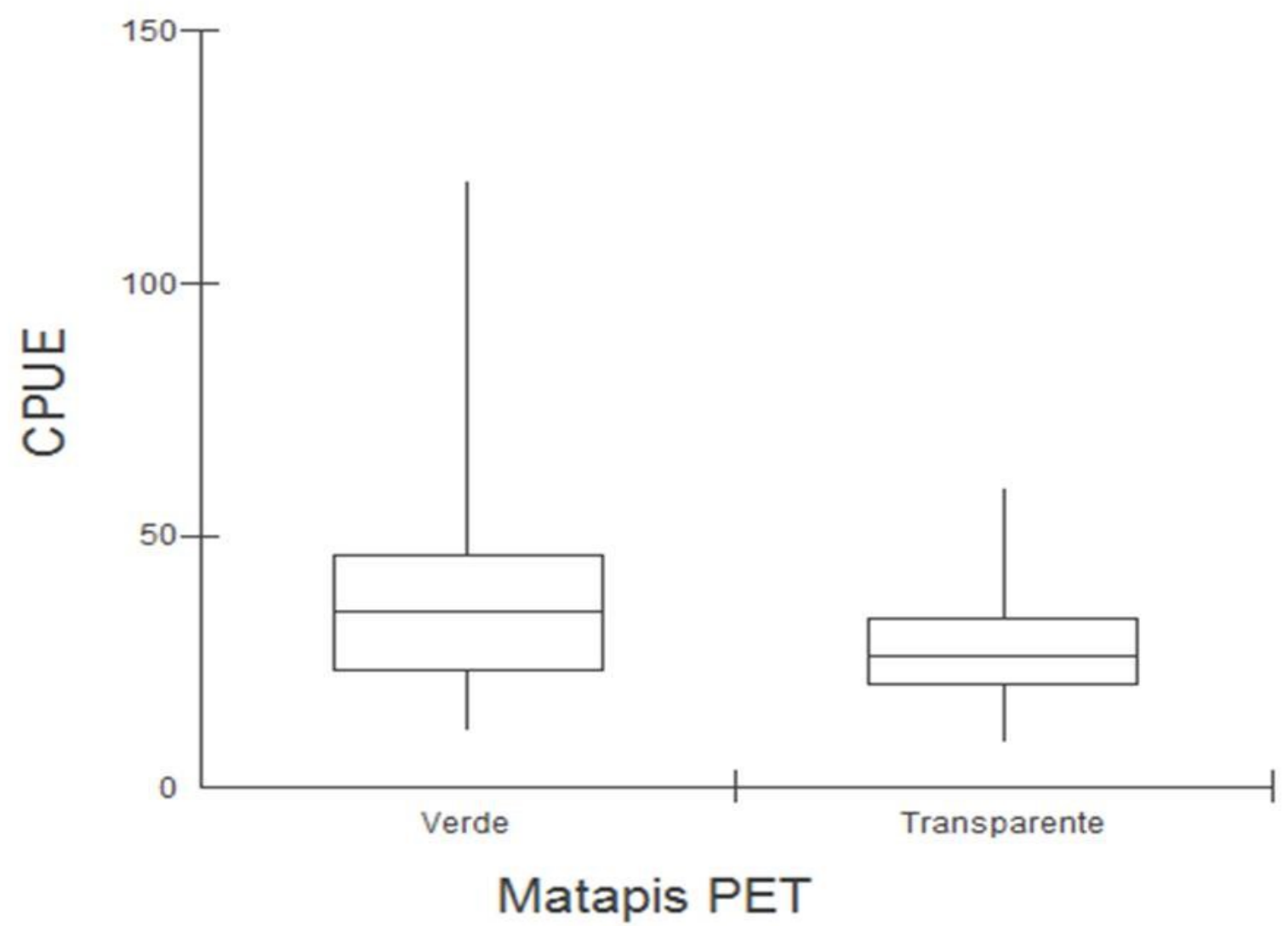

Figura 4. Variação da CPUE dos matapis PET (verde e transparente) sobre os camarões.

\section{CONCLUSÕES}

As armadilhas matapi PET de cor verde foram mais eficientes na captura de camarões do gênero Macrobrachium, do que as armadilhas matapi PET transparentes com a mesma CPUE, possivelmente por favorecer o bem-estar dos animais.

Os matapis de garrafas PET são econômicos, práticos e ecologicamente viáveis para os pesquisadores, pescadores e produtores. São de fácil confecção, além de sua vida útil ser maior que o do matapi feito de jupati. A reutilização de garrafas PET ajuda na diminuição de descarte do material no meio ambiente, garantindo a sustentabilidade do ecossistema.

Desta forma, experiências adicionais, como o estudo de outros fatores operacionais sobre as capturas durante o dia e a noite, sobre os efeitos das interações interespecíficas sobre as capturas, sobre a influência de ambientes lênticos e lóticos nas capturas permitiriam uma melhor compreensão do funcionamento dessas armadilhas.

\section{AGRADECIMENTOS}

Aos analistas Willyam Stern Porto, Carlos Eduardo D'Alencar Mendonça, e aos alunos do LOAM pelo apoio durante as coletas.

\section{REFERÊNCIAS BIBLIOGRÁFICAS}

Aquino-Pereira, S.L., Quadros, M., Porto, W.S. \& Santos, A.N.A. 2013. Características da piscicultura no município de Mucajaí - Roraima. In: Congresso Brasileiro de Aquicultura de Espécies Nativas, 4., 2013, Belém. Resusmos... Belém, PA: Aquabio.

Ayres, M., Ayres-Jr, M., Ayres, D.L. \& Santos, A.A.S. 2007. Bioestat: aplicações estatísticas nas áreas das Ciências Biomédicas. Versão 5.0. Belém, Pará: Sociedade Civil Mamirauá, MCT-CNPq, 324 p.

Camargo, M., Leão, J., Oliveira, A., Márcio, A., Silva, E., Acácio, F., Silva, G., Sampaio, L., Viana, M., Antunes, T. \& Silva, W. 2009. Matapi pet: uma nova proposta para a exploração sustentável do camarão amazônico Macrobrachium amazonicum (Heller, 1862). UAKARI 5 1: 91-96.

Castro, P. M. de. \& Silva, M. R. da. 2013. Caranguejos e Camarões (Crustacea: Decapoda) do Igarapé do Perdido, Apiaú, Mucajaí, Roraima. Boletim do 
Perdido, Apiaú, Mucajaí, Roraima. Boletim do Museu Integrado de Roraima, 7 (1): 40-44.

Castro, P. M. de. 2012. A coleção de crustáceos do Museu Integrado de Roraima (MIRR). Anais do VI Congresso Brasileiro sobre Curstáceos, pg. 51.

Cavalcante, R. M. \& Castro, P.M. 2014. Verificação das Potencialidades do Camarão de água doce Macrobrachium jelskii (Miers, 1877) com o cultivo em viveiros de tambaqui Colossoma macropomum no Município de Alto Alegre no Estado de Roraima. Revista Eletrônica, Ambiente, Gestão e Desenvolvimento. 6: 45-51.

Fouilland, E. \& Fossati, O. 1996. Trapping efficiency of plastic bottle 'wickertraps' for population assessment of river Macrobrachium (Crustacea: Decapoda). Fisheries Research 28: 343-351.

Fouilland, E. \& Fossati, O. 1998. Effects of some operational factors on Macrobrachium (Decapoda, Palaemonidae) sampling using small 'wickertraps'. Fisheries Research 34: 87-92.

Guterres, P.M.C., Santos, M.A.L., Costa, F.Z., Silva, J.T. \& Lima, D.A. 2010. Diversidade de crustáceos dacápodas em igarapé localizado em área antropizada, Mucajaí, Roraima. Resumos da Reunião Regional da SBPC em Boa Vista. pg. 1245. (http:// www.sbpenet.org.br/livro/boavista/ resumos/1295.htm). Acesso em 15/12/2016.

Lima, D.A. \& Castro, P.M. de. 2012. Reprodução de Macrobrachium amazonicum (Heller, 1862) (Crustacea: Decapoda: Palaemonidae) em viveiro de piscicultura na região do Apiaú, Mucajaí, Roraima. Anais do VI Congresso Brasileiro sobre Curstáceos, pg. 117.

Lima, D.A., Zanetti, F.C., Castro, P.M., Santos, M.A.L. \& Rodrigues, M. 2012. Camarões paleomonídeos em igarapés de florestas semidecíduais no Mucajaí, Roraima. Anais do VI Congresso Brasileiro sobre Curstáceos, pg. 130.

Lin, X. \& Omori, M. 1993. Effect of tank colouration on the feeding rates of zoeal larvae of the giant freshwater shrimp Macrobrachium rosenbergii. Bulletin of the Plankton Society of Japan. 40: 19-25.

Luchiari, A. C. \& Pirhonen, J. 2008. Effects of ambient colour on colour preference and growth of juvenile rainbow trout Oncorhynchus mykiss (Walbaum). Journal of Fish Biology. 72: 1504-1514.

Luchiari, A. C., Freire, F. A. M., Pirhonen, J. \& Koskela, J. 2009. Longer wavelengths of light improve the growth, intake and feed efficiency of individually reared juvenile pikeperch Sander lucioperca (L.). Aquaculture Research. 40: 880-886.

Mariappan, P. \& Balasundaram, C. 1999. Prevalence of limb loss in Macrobrachium nobilii (Decapoda: Caridea). Indian Journal Fish. 46:61-66.

Marshall, N.J., Jones, J.P. \& Cronin, T.W. 1996.
Behavioural evidence for color vision in stomatopod crustaceans. Journal of Comparative Physiology A, 179: 473-481.

Meager, J.J., Williamson, I., Loneragan, N.R. \& Vance, D.J. 2005. Habitat selection of juvenile banana prawns, Penaeus merguiensis de Man: Testing the roles of habitat structure, predators, light phase and prawn size. Journal of Experimental Marine Biology and Ecology. 324: 89-98.

New, M.B., D'Abramo, L.R., Valenti, W.C. \& Singholka, S. 2000. Sustainability of freshwater prawn culture. In: NEW, M.B. \& VALENTI, W.C. (Ed.) Freshwater prawn culture: the farming of Macrobrachium rosenbergii. Oxford, Blackwell Science. p. 429-443.

Santos, D.B. dos. 2013. Comportamento e desempenho do camarão de água doce Macrobrachium rosenbergii (De Man, 1879) em cultivos misto e monossexo. Tese de Doutorado, Universidade Federal do Rio Grande do Norte - UFRN, Natal. $141 \mathrm{p}$.

Valenti, W. C. 2002. Criação de camarões de água doce. In: Congresso de Zootecnia, 12o, Vila Real, Portugal: Associação Portuguesa dos Engenheiros Zootécnicos. Anais... p. 229-237.

Vieira, I.M. 2003. Bioecologia e pesca do camarão, Macrobrachium amazonicum (Heller, 1862) no baixo Rio Amazonas - AP. Dissertação de Mestrado, Universidade Federal do Amapá, Macapá. 153p.

Volpato, G.L., Duarte, C.R.A. \& Luchiari, A.C. 2004. Environmental color affects Nile tilapia reproduction. Brazilian Journal of Medical and Biological Research. 37: 479-483.

Yasharian, D., Coyle, S.D., Tidwell, J.H., Stiwell, W.E. 2005. The effect of tank colouration on survival, metamorphosis rate, growth and time to metamorphosis freshwater prawn (Macrobrachium rosenbergii) rearing. Aquaculture Research . 36: 278 -283 .

Zar, J.H. 2010. Biostatistical analysis. 5 ed., PrenticeHall Inc., Upper Saddle River, New Jersey. 943p. 